\title{
(จ) Surgical assessment of the insula. Part 1: surgical anatomy and morphometric analysis of the transsylvian and transcortical approaches to the insula
}

\author{
Arnau Benet, MD, Shawn L. Hervey-Jumper, MD, Jose Juan González Sánchez, MD, PhD, \\ Michael T. Lawton, MD, and Mitchel S. Berger, MD \\ Department of Neurosurgery, University of California, San Francisco, California
}

OBJECTIVE Transcortical and transsylvian corridors have been previously described as the main surgical approaches to the insula, but there is insufficient evidence to support one approach versus the other. The authors performed a cadaveric comparative study regarding insular exposure, surgical window and freedom, between the transcortical and transsylvian approaches (with and without cutting superficial sylvian bridging veins). Surgical anatomy and skull surface reference points to the different insular regions are also described.

METHODS Sixteen cadaveric specimens were embalmed with a customized formula to enhance neurosurgical simulation. Two different blocks were defined in the study: first, transsylvian without (TS) and with the superficial sylvian bridging veins cut (TSVC) and transcortical (TC) approaches to the insula were simulated in all (16) specimens. Insular surface exposure, surgical window and surgical freedom were calculated for each procedure and related to the BergerSanai insular glioma classification (Zones I-IV) in 10 specimens. Second, the venous drainage pattern and anatomical landmarks considered critical for surgical planning were studied in all specimens.

RESULTS In the insular Zone I (anterior-superior), the TC approach provided the best insular exposure compared with both TS and TSVC. The surgical window obtained with the TC approach was also larger than that obtained with the TS. The TC approach provided 137\% more surgical freedom than the TS approach. Only the TC corridor provided complete insular exposure. In Zone II (posterior-superior), results depended on the degree of opercular resection. Without resection of the precentral gyrus in the operculum, insula exposure, surgical windows and surgical freedom were equivalent. If the opercular cortex was resected, the insula exposure and surgical freedom obtained through the TC approach was greater to that of the other groups. In Zone III (posterior-inferior), the TC approach provided better surgical exposure than the TS, yet similar to the TSVC. The TC approach provided the best insular exposure, surgical window, and surgical freedom if components of Heschl's gyrus were resected. In Zone IV (anterior-inferior), the TC corridor provided better exposure than both the TS and the TSVC. The surgical window was equivalent. Surgical freedom provided by the TC was greater than the TS approach. This zone was completely exposed only with the TC approach. A dominant anterior venous drainage was found in $87 \%$ of the specimens. In this group, $50 \%$ of the specimens had good alternative venous drainage. The sylvian fissure corresponded to the superior segment of the squamosal suture in 14 of 16 specimens. The foramen of Monro was $1.9 \mathrm{~cm}$ anterior and $4.42 \mathrm{~cm}$ superior to the external acoustic meatus. The $\mathrm{M}_{2}$ branch over the central sulcus of the insula became the precentral $\mathrm{M}_{4}$ (rolandic) artery in all specimens.

CONCLUSIONS Overall, the TC approach to the insula provided better insula exposure and surgical freedom compared with the TS and the TSVC. Cortical and subcortical mapping is critical during the TC approach to the posterior zones (II and III), as the facial motor and somatosensory functions (Zone II) and language areas (Zone III) may be involved. The evidence provided in this study may help the neurosurgeon when approaching insular gliomas to achieve a greater extent of tumor resection via an optimal exposure.

http://thejns.org/doi/abs/10.3171/2014.12.JNS142182

KEY WORDS insula; glioma; transsylvian approach; transcortical approach; brain tumor; sylvian fissure; oncology; anatomy 
$\mathrm{I}$ NSULAR gliomas are among the most challenging lesions to manage in neurosurgery. In contrast to other regions of the cerebral cortex, the insular lobe is located beyond the cerebral surface, in the depth of the sylvian fissure and covered by the opercula and many critical vascular structures. Additionally, a fair amount of cortical areas covering the insula are functional. Also, the venous complex covering the sylvian fissure often contains important drainage that must be preserved, further narrowing surgical options for tumor removal. Despite this challenging situation, there is evidence that the extent of tumor resection greatly impacts survival in patients with insular gliomas. ${ }^{3,6,13,17}$ Therefore, although surgically complex, neurosurgeons should be able to aggressively, yet safely, resect these tumors.

Insular gliomas were initially accessed through the sylvian fissure via the transsylvian approach, as previously described by Yaşargil and further developed in the last 2 decades. 5,9,20,21 The transsylvian fissure approach requires wide opening of the superficial and deep sylvian cisterns and careful protection of the opercular arteries and their perforators, as well as preservation of the dominant superficial sylvian veins. The transsylvian approach to the insula requires opercular retraction, which is often limited by the superficial sylvian veins bridging the sylvian fissure. Thus, for larger insular lesions, this approach will not yield optimal surgical access to achieve the desired surgical results.

With the use of cortical and subcortical electrical stimulation, the lateral surface of the opercula may be mapped during an awake procedure, allowing identification and preservation of functional areas. Removing silent cortical areas such as the operculum and superior temporal gyrus is an emerging strategy to maximize the extent of tumor resection while preserving the superficial vascular structures. ${ }^{4}$ We have previously reported our transcortical "window" technique and developed an anatomical division of the insula that enabled a preoperative prediction for extent of resection. ${ }^{13}$ Nevertheless, it is often the experience of the surgeon, rather than the rationale to enhance exposure of the insula, that determines which approach (i.e., transsylvian or transcortical) is optimal.

At present, there are no supportive data based on cadaveric surgical simulation to determine the differences in surgical access to the insula between the transsylvian (TS) and transcortical (TC) approach. Furthermore, there is a lack of evidence as to which technique or a combination of the two would yield the optimal surgical window to maximize the extent of resection safely.

In this study, we assessed the surgical profile (i.e., insula exposure, surgical window, and surgical freedom) of the TS and TC approaches to the insula using a cadaveric surgical simulation model. Using a sequential experimental design, we asked whether there is a significant difference in insular exposure, surgical window, and surgical freedom (Fig. 1) between the following approaches: the TS approach, the TS after cutting the superficial sylvian veins bridging over the sylvian fissure (TSVC), and the TC approach. Also, we sought to evaluate the venous drainage of the perisylvian region to provide evidence on the likelihood of venous dominancy as a limitation for venous sac-

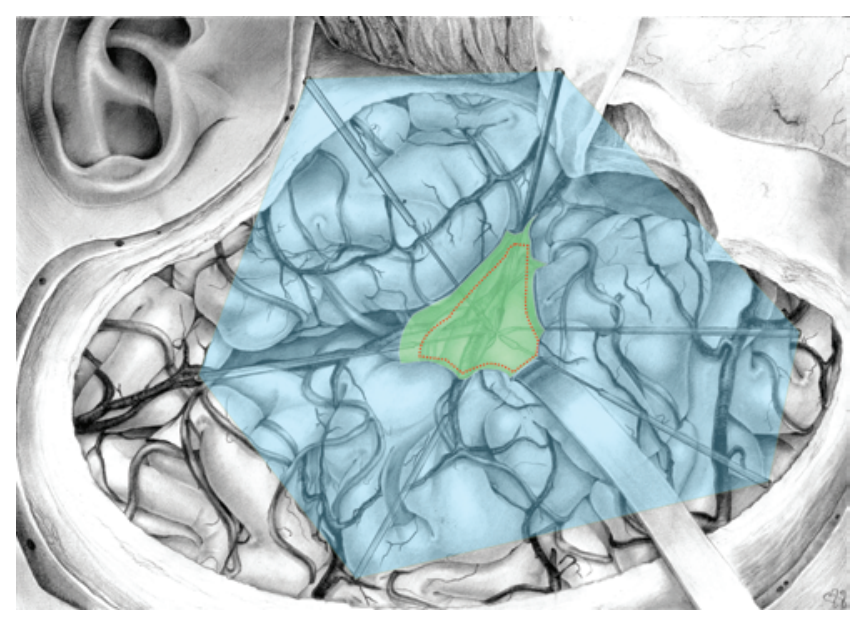

FIG. 1. Illustration of the concepts of insular exposure and surgical window and freedom. The left transsylvian approach to insular Zone I was conceptualized by the medical illustrator. The insular exposure (dotted shape) is the area over the insular cortex available through a surgical approach. The insular exposure is the amount of access to the insular cortex provided by each approach. The surgical window (green [shaded area in center of figure]) is the area existing between the neurovascular structures limiting the space to access the insula, i.e., the corridor. The surgical window provides information on the space available to pass instruments to a particular insular zone by each approach. The surgical freedom (blue [outer shape]) is the area formed by the top of a dissector that, while pivoting on a surgical landmark, contacts the perimeter of the surgical opening. The surgical freedom provides a measure of the degree of maneuverability or ease of manipulating instruments to a particular point in the insula. Copyright Arnau Benet. Published with permission. Figure is available in color online only.

rifice during a TS approach. Additionally, we studied the surgical anatomy related to each procedure along with the final surface exposure of the insula. Finally, we describe 2 skull surface reference points to infer the position of the insular zones.

\section{Methods \\ Study Design}

To study and compare the surgical corridors of the transsylvian (TS), transsylvian with bridging veins cut (TSVC), and transcortical (TC) approaches (independent variables) to the insula, an experimental laboratory investigation was designed. Measurements included insular exposure, surgical window, and surgical freedom (dependent variables) resulting from each approach in 10 specimens (all continuous ratio variables). Additionally, we carried out a descriptive study in 16 specimens, providing critical information for the surgical planning and approach selection process. The descriptive study included categorical dichotomous variables, such as the presence of bridging veins over the sylvian fissure, dominance of the superficial sylvian vein complex, presence of vein clustering, continuity from the artery of the central sulcus of the insula to the rolandic artery, and the relationship between the superior segment of the squamosal suture and the sylvian fissure. Additionally, the number of $\mathrm{M}_{2}, \mathrm{M}_{3}$, and $\mathrm{M}_{4}$ (continuous interval variables), the distance from the external acoustic meatus to the foramen of Monro (anterior-posterior and cranial-caudal), and the distance from the temporal pole 
to the cortical resection margin (continuous ratio variables) were recorded.

\section{Insula Surgical Classification}

To facilitate data interpretation and enhance the surgical relevance of this study, the Berger-Sanai surgical classification scheme of the insula was used to subdivide the insula into 4 zones. ${ }^{13}$ Therefore, the insula was divided into anterior and posterior from the axial projection of the foramen of Monro; and superior to inferior by the sylvian fissure line projected over the insular cortex (Fig. 2).

\section{Description of the Variables \\ Independent Variables}

The TS approach utilizes only the sylvian fissure split and retraction over the opercula to expose the insula. In this approach, the superficial and deep sylvian cisterns are opened widely throughout the fissure. The TSVC allows additional retraction to the TS by cutting the bridging veins crossing the sylvian fissure. The TC approach uses different degrees of cortical resection to expose the

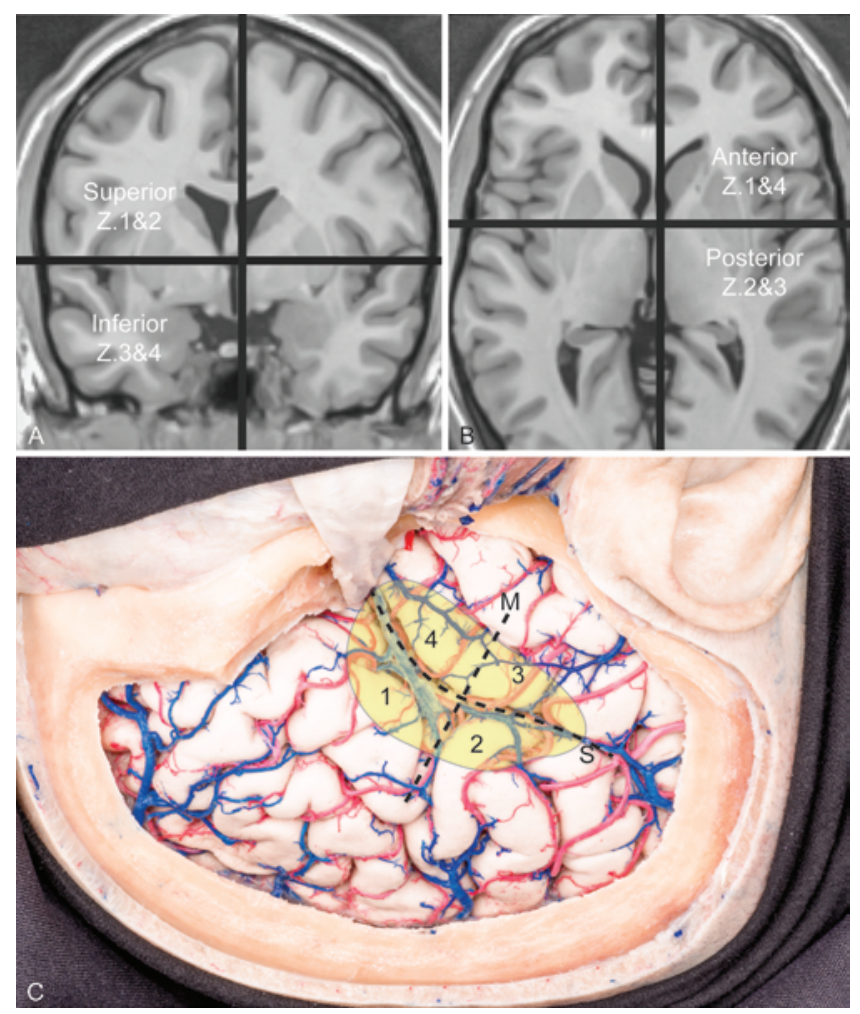

FIG. 2. Anatomical classification of the insula. A T1-weighted MR image was obtained from each specimen, and the foramen of Monro was identified in each volume. A linked radiological display was used to identify the projected point of the foramen of Monro in the insula cortex (A-C). In the coronal view (A), the insula is divided into superior and inferior halves by the sylvian line-the sylvian fissure projected to the insular cortex. In the axial view (B), the projection of the foramen of Monro was used to divide the insula into anterior and posterior halves. A photograph of the intradural phase of a right pterional approach was taken to superimpose the insula (yellow label) on the cortical surface (C). The line passing through the foramen of Monro and the line projected from the sylvian fissure divide the insula in 4 zones. $M=$ projected line of the foramen of Monro; $S$ = projected line of the sylvian fissure. insula. In the TC, the bridging veins are preserved and the space to access the insula results from multiple windows between the middle cerebral arteries and the superficial sylvian veins. In Zones 2 and 3, the TC was further divided into two subcategories. In Zone II, the first set of dependent variables was taken before and after the operculum portion of the precentral gyrus was resected. In Zone III, the first set of dependent variables was taken before and after removing Heschl's gyrus. This subcategorization was set in the experimental design to include the option for a less invasive cortical resection and to investigate the significance that resecting these areas may have during a TC approach.

\section{Dependent Variables}

The insula exposure is the area (in $\mathrm{cm}^{2}$ ) of insular cortex exposed and surgically reachable for bimanual dissection. This area was obtained by touching the insular cortex with the navigation probe to obtain the stereotactic coordinates as previously described. ${ }^{1}$ The surgical window is a polygonal area formed by the structures that, in more superficial planes, limit the corridor to the insula exposure. Typical structures limiting the surgical window were arteries, veins and cerebral cortex. The surgical window area was obtained by touching the limiting structures of the corridor with the navigation. The surgical freedom provides an objective indication on how freely an instrument can be moved in relation to a particular target. In our study, the surgical freedom $\left(\mathrm{cm}^{2}\right)$ was targeted to a point in the center of each insular zone. A Rhoton No. 5 dissector was pivoted to a target selected at the center of each insular zone and its handle moved in the perimeter of the surgical window to capture the contour of the corridor. The navigation probe touched the tip of the dissector handle in each major change in trajectory and recorded a set of stereotactic coordinates. ${ }^{1}$ Additionally, we measured the extent of cortical resection after a transcortical approach to each insular zone. Three stereotactic points were obtained in each zone by touching the lips of the sylvian fissure with the navigation probe. After the resection was complete for each zone, the same points were touched with the navigation probe over the resection rim. Three linear measurements were obtained by calculating the distance between each point obtained over the lip of the sylvian fissure (preresection) and its equivalent at the resection margin (postresection).

\section{Specimen Preparation}

We included 16 embalmed human cadaveric specimens from donors without previous history of head and neck pathology and with a postmortem window of 72 hours. The specimens were embalmed with our customized formula for neurosurgical simulation and prepared for surgical research as described by our group. ${ }^{1}$ A 3-T T1-weighted MR images were acquired from all specimens before the study. Radiological data were uploaded to the navigation system (Stryker NAV3) and registered to the specimen before each experiment.

\section{Experiment Design}

The specimen was positioned for a pterional approach 
to the insula and rigidly attached to a surgical table. After exposure of the skull, the foramen of Monro was identified with the aid of navigation and measurements referenced to the external acoustic meatus were taken. The squamosal suture was touched with the navigation probe to identify the relationship to the sylvian fissure, which in turn was marked on the skull. A wide pterional craniotomy was designed based on the foramen of Monro, the sylvian fissure (already marked in the skull) and the opercula including at least $3 \mathrm{~cm}$ of extra cortical surface to allow retraction. The dura mater was incised and reflected anteriorly over the sphenoid ridge.

The sylvian fissure was completely split as previously described..$^{12}$ All venous channels were carefully dissected and preserved. Dynamic retraction was applied to each zone's operculum following the navigation probe as the stereotactic measurements were taken. The amount of retraction, which was measured in length, was the maximum allowed by the bridging veins or before cortical damage. The arterial and venous cortical systems were manually drawn separately in a printed hemispheric template map. The number, size, and trajectory of the veins were recorded as well.

Next, the veins crossing the sylvian fissure were marked and cut. All dependent variables were measured again in the zones previously limited by bridging veins. Following this, the veins previously cut were anastomosed using 8-0 sutures and a Lawton bypass set (Mizuho America).

After restoring the venous system completely, the TC approach was started. The corticotomy was performed using microsurgical instruments and magnified dissection under the surgical microscope (Carl Zeiss Pentero), with caution taken to preserve $\mathrm{M}_{3}$ and $\mathrm{M}_{4}$ arteries transitioning to the cortical surface. Multiple arterial-venous windows were generated by the end of each corticotomy. All dependent variables were measured at this point, including the TC subdivision into Zone II with and without the precentral motor cortex and Zone III with and without Heschl's gyrus. Reference pins were set along the margins of the corticotomy to guide the transition to the next zone. The cortical limits after the corticotomy were drawn into the hemispheric template map to track the extent of cortical resection. The distances between the cortical resection margin and both the operculum and the temporal pole were also taken at this point. Finally, the insular vasculature was also drawn.

\section{Statistical Analysis}

All data collected in this study were entered in a spreadsheet that was uploaded into statistical software (JMP v. 11.0, SAS institute) for statistical processing. Unpaired Student t-tests were calculated on the dependent continuous variables to determine significance between the compared variables and groups. A p value of 0.05 was considered significant. The mean and standard deviation for continuous variables and percentages for continuous and categorical variables were also calculated from the spreadsheet.

\section{Results}

Data collected in the present study include quantitative analysis of surgical variables (i.e., insular exposure, surgical window, and surgical freedom) for each approach as well as a descriptive analysis of the surgical anatomy of the transsylvian and transcortical approaches to the insula.

\section{Morphometric Assessment}

To ease data interpretation, insular exposure and surgical freedom for each approach were grouped and provided for each insular zone (Figs. 3 and 4).

\section{Zone I}

The TC corridor provided the best insular exposure in Zone I compared with both TS $(4.62 \pm 0.6$ [SD] vs 7.21 $\left.\pm 0.8 \mathrm{~cm}^{2}, \mathrm{p}<0.05\right)$ and TSVC $(5.21 \pm 0.98$ vs $7.21 \pm 0.8$ $\left.\mathrm{cm}^{2}, \mathrm{p}<0.05\right)$. Even with the veins cut, the TC approach provided $140 \%$ more insula exposure than the TS. Also, cutting the bridging veins in Zone I did not provide a significant increase in either the final insula exposure or the
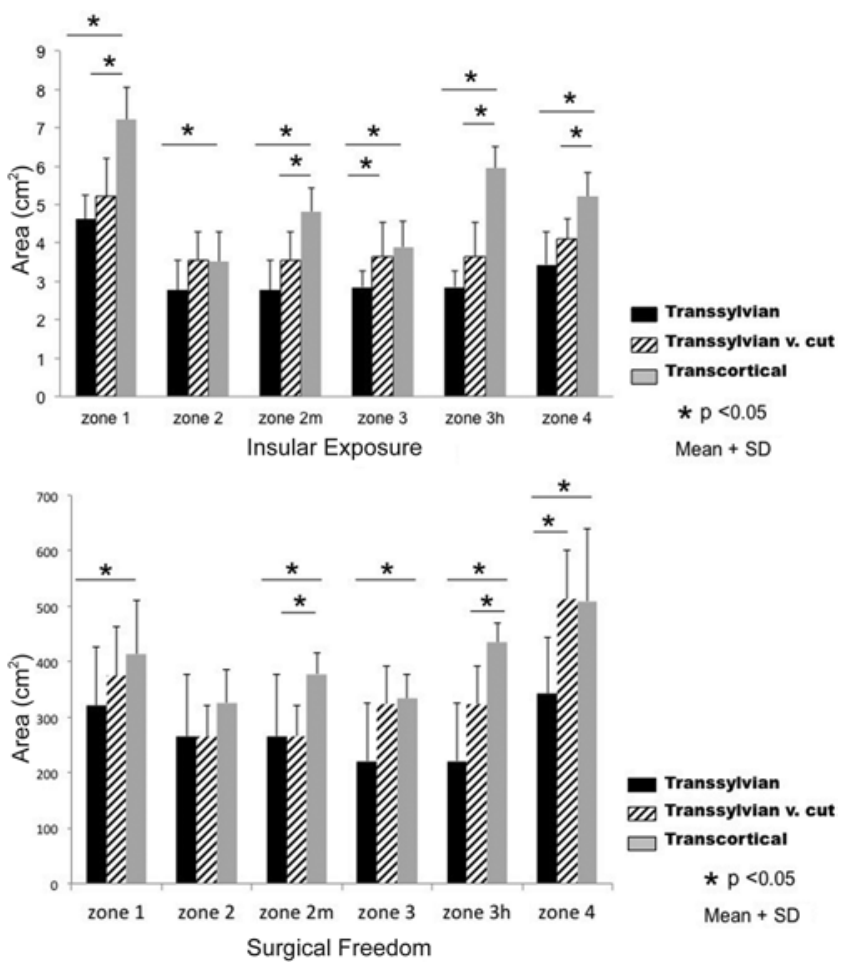

FIG. 3. Graph of the statistical analysis of the insular exposure (upper) and surgical freedom (lower) obtained during the transsylvian approach (TS), transsylvian approach with bridging veins cut (TSVC) and transcortical approach (TC). The mean, standard deviation (error bars), and statistical significance of the difference in insular exposure (upper) or surgical freedom (lower) for the TS, TSVC, and TC approaches to the insula are shown for each zone. The TC approach provides more insular exposure than the TS and TSVC except in Zone II. Only when the opercular rim of the precentral gyrus was resected did the TC approach provide more exposure than the TSVC. The maximum difference in insular exposure was found in Zone I and IIIh. Overall, surgical freedom is smallest in the TS group and largest in the TC group, except in Zone IV. Surgical freedom obtained during a complete TC approach was significantly greater than in the TSCV. Cutting the bridging veins provided greater surgical freedom in Zone IV only. v. = bridging veins; Zone IIm = Zone II where the opercular rim of the precentral gyrus was resected during the transcortical approach; Zone IIlh = Zone III where Heschl's gyrus was partially resected during the transcortical approach. 


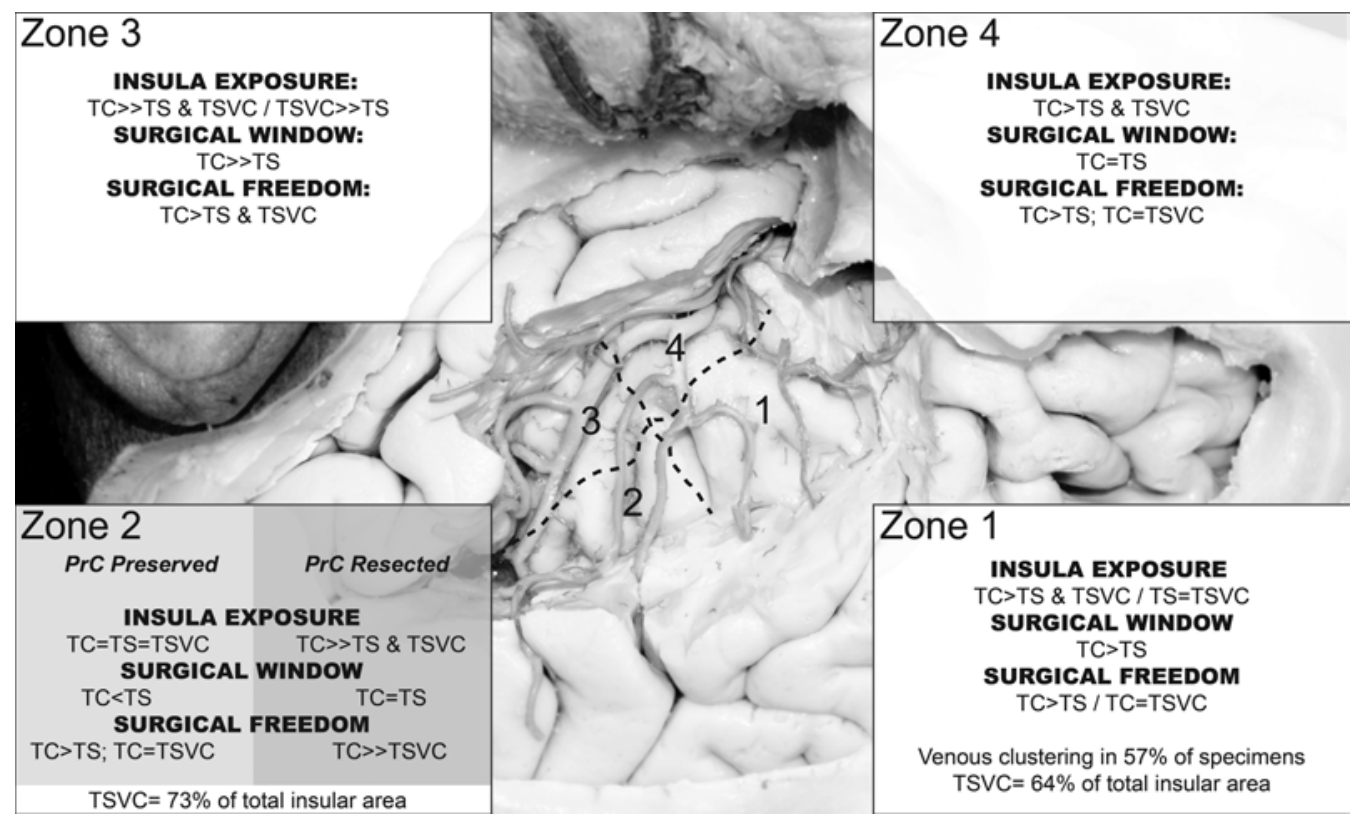

FIG. 4. Summary of the comparative analysis between the TS, TSVC, and TC approaches to each zone of the left insula. A photograph of the surgical simulation after a left TC approach was taken and used to illustrate the division of the insular cortex into 4 zones according to the Berger-Sanai classification of the insula. The sylvian line (anterior-posterior dashed line) divides the insula into ventral and dorsal parts. The Monro foramen and its lateral projection (superior-inferior dashed line) divide the insula into rostral and caudal parts. Zone II is further divided into 2 groups depending on the degree of frontoparietal opercula corticotomy during the TC approach. In Zone III, the TC group includes partial resection of Heschl's gyrus. All results included in the vignettes are statistically significant. $\mathrm{PrC}=$ precentral gyrus.

surgical freedom when the TS was used. The mean surgical window obtained in the TC was larger than in the TS $\left(6.18 \pm 0.6\right.$ vs $\left.9.65 \pm 1.7 \mathrm{~cm}^{2}, \mathrm{p}<0.05\right)$. The maximal retraction length at Zone I was $1 \pm 3 \mathrm{~cm}$. Cortical resection for total exposure of Zone I was $1.5 \pm 0.5 \mathrm{~cm}$ at the inferior frontal gyrus. Although the TC provided a significant increase in surgical freedom compared with the TS $(137 \%$, $\mathrm{p}<0.05)$, there was no statistically significant difference in comparison with the TSVC.

\section{Zone II}

Results obtained in Zone II were dependent on the degree of the corticotomy. When the precentral gyrus was preserved during the TC dissection, the insular exposure, surgical window, and surgical freedom were equivalent to that of the TSVC. The mean insular exposure in the TC group was greater than the TS $(2.76 \pm 0.8$ vs $3.51 \pm 0.8$ $\left.\mathrm{cm}^{2}, \mathrm{p}<0.05\right)$ but similar to that of the TSVC $(3.56 \pm 0.7$ vs $3.51 \pm 0.8 \mathrm{~cm}^{2}, \mathrm{p}=0.9$ ). There was no statistically significant difference in insular exposure between the TS and the TSVC, thus cutting the bridging veins was not advantageous in Zone II. The mean surgical window obtained in the TS (obtained by applying maximal retraction along the opercular lip at the inferior parietal lobule) was greater than that of the TC (with the precentral gyrus preserved) $\left(4.43 \pm 1.1\right.$ vs $\left.3.42 \pm 0.7 \mathrm{~cm}^{2}, \mathrm{p}<0.05\right)$. The maximal retraction length at Zone I was $1.2 \pm 2 \mathrm{~cm}$. There were no differences in surgical freedom between the TS, TSVC, and TC.

On the other hand, when the opercular rim of the precentral gyrus was removed during the TC dissection, there was a clear advantage on the TC over all other groups.
Specifically, the insula exposure obtained in the TC was $146 \%$ that of the TSVC $(\mathrm{p}<0.05)$. Also, the surgical windows obtained during the TC and TS were similar (5.08 \pm 0.8 vs $4.43 \pm 1.1 \mathrm{~cm}^{2}, \mathrm{p}=0.16$ ). Moreover, the mean surgical freedom obtained in the TC was $171 \%$ that of the TSVC $(p<0.05)$. Complete TC exposure of the insular cortex at Zone II required excision of the inferior $1.2 \pm 0.2 \mathrm{~cm}$ of the precentral and postcentral gyri, which completely exposed the superior peri-insular sulcus, the anterior and posterior long gyri and Heschl's gyrus in the temporal operculum.

\section{Zone III}

In Zone III, The TC approach provided better surgical exposure than the TS $\left(3.89 \pm 0.6\right.$ vs $2.85 \pm 0.4 \mathrm{~cm}^{2}$, $\mathrm{p}<0.05)$. However, if Heschl's gyrus was removed, the insula exposure of the TC was superior to that of the TSVC. In this zone, cutting the bridging veins during the TS increased insular exposure substantially $(3.64 \pm 0.8 \mathrm{vs}$ $2.85 \pm 0.4 \mathrm{~cm}^{2}, \mathrm{p}<0.05$ ). Resecting Heschl's gyrus provided $156 \%$ of the insula exposure obtained during a TC approach. Also, when the TC included resection of Heschl's gyrus, the insular exposure obtained was $164 \%$ that of TSVC, which was a statistically significant increase ( $p$ $<0.05$ ). When Heschl's gyrus was resected during TC, the surgical window was $176 \%$ that of the TS $(p<0.05)$. The surgical freedom obtained in the TC group was greater than that of the TS group $\left(334.16 \pm 43\right.$ vs $221.14 \pm 105 \mathrm{~cm}^{2}$, $p<0.05)$ but similar to that of the TSVC $(p=0.75)$. However, after resection of Heschl's gyrus, the surgical freedom obtained in the TC was greater than in either of the other groups $(\mathrm{p}<0.05)$. Cutting the bridging veins during 
the TS approach did not increase the surgical freedom ( $\mathrm{p}$ $=0.09$ ). The maximal retraction length at Zone III was $1 \pm$ $2 \mathrm{~cm}$. Cortical resection for complete exposure of Zone III was $1.3 \pm 0.3 \mathrm{~cm}$ of the superior temporal gyrus.

\section{Zone IV}

The TC corridor provided greater insular exposure than both the TS $\left(5.2 \pm 0.6\right.$ vs $\left.3.44 \pm 0.8 \mathrm{~cm}^{2}, \mathrm{p}<0.05\right)$ and the TSVC $\left(5.2 \pm 0.6\right.$ vs $\left.4.11 \pm 0.5 \mathrm{~cm}^{2}, \mathrm{p}<0.05\right)$. Cutting the veins during the TS approach did not increase insula exposure (TSVC $4.11 \pm 0.5$ vs TS $3.44 \pm 0.8 \mathrm{~cm}^{2}, \mathrm{p}=0.08$ ). There was no difference between the TC and the TS with respect to surgical window $\left(5.96 \pm 1.1\right.$ vs $5.38 \pm 1.2 \mathrm{~cm}^{2}$, $\mathrm{p}=0.28$ ). The TC provided greater surgical freedom than the TS $\left(508.8 \pm 131\right.$ vs $\left.341.7 \pm 101 \mathrm{~cm}^{2}, \mathrm{p}<0.05\right)$, but equivalent to that of the TSVC $(508.8 \pm 131$ vs $513.14 \pm$ $\left.87 \mathrm{~cm}^{2}, \mathrm{p}<0.05\right)$. The TSVC provided greater surgical freedom than the TS $\left(513.14 \pm 87\right.$ vs $341.76 \pm 101 \mathrm{~cm}^{2}, \mathrm{p}<$ 0.05 ). The maximal retraction length at Zone IV was $1 \pm 3$ $\mathrm{cm}$. Complete exposure of the inferior peri-insular sulcus required resection of the entire width of the superior temporal gyrus from the foramen of Monro anteriorly to $1.5 \pm$ $0.6 \mathrm{~cm}$ posterior to the temporal pole.

\section{Surgical Anatomy}

In Zone I, the TS corridor provided exposure of the insular apex and the sylvian line, and only the TC pro- vided exposure of the superior peri-insular sulci (Fig. 5). The TSVC provided exposure to $64 \%$ of Zone I, which included the proximal portion of the anterior, middle and posterior short gyri as well as the apical portion of the accessory gyrus. In $57 \%$ of cases, a vein cluster arising from the prefrontal cortex severely limited exposure to the middle short gyrus and the posterior half of the anterior short gyrus. When the TSVC was used, the middle short gyrus of the insula was better accessed, as the main bridging veins cross the sylvian fissure at this region. However, it did not provide a significant increase in surgical exposure. Only the TC corridor allowed complete exposure of the superior peri-insular sulcus and the entire area of the anterior, middle, posterior and accessory insular gyri in Zone I. Moreover, if the surgical table was tilted to the ipsilateral side and the head turned down $15^{\circ}$ (by either increasing Trendelenburg or tilting the back rest down), the Eberstaller gyrus and the lateral lenticulostriate arteries were also exposed. The corticotomy necessary to reach Zone I completely required excision of $60 \%$ of the pars orbicularis and opercularis, and $20 \%$ of the pars triangularis.

In Zone II, the TS corridor provided insular exposure limited to the sylvian line whereas the TC corridor allowed greater exposure of the posterior long gyrus and the anterior long gyrus. The TS corridor was severely limited by the narrow shape of the posterior half of the sylvian fissure (Fig. 6). Even when the bridging veins were cut dur-
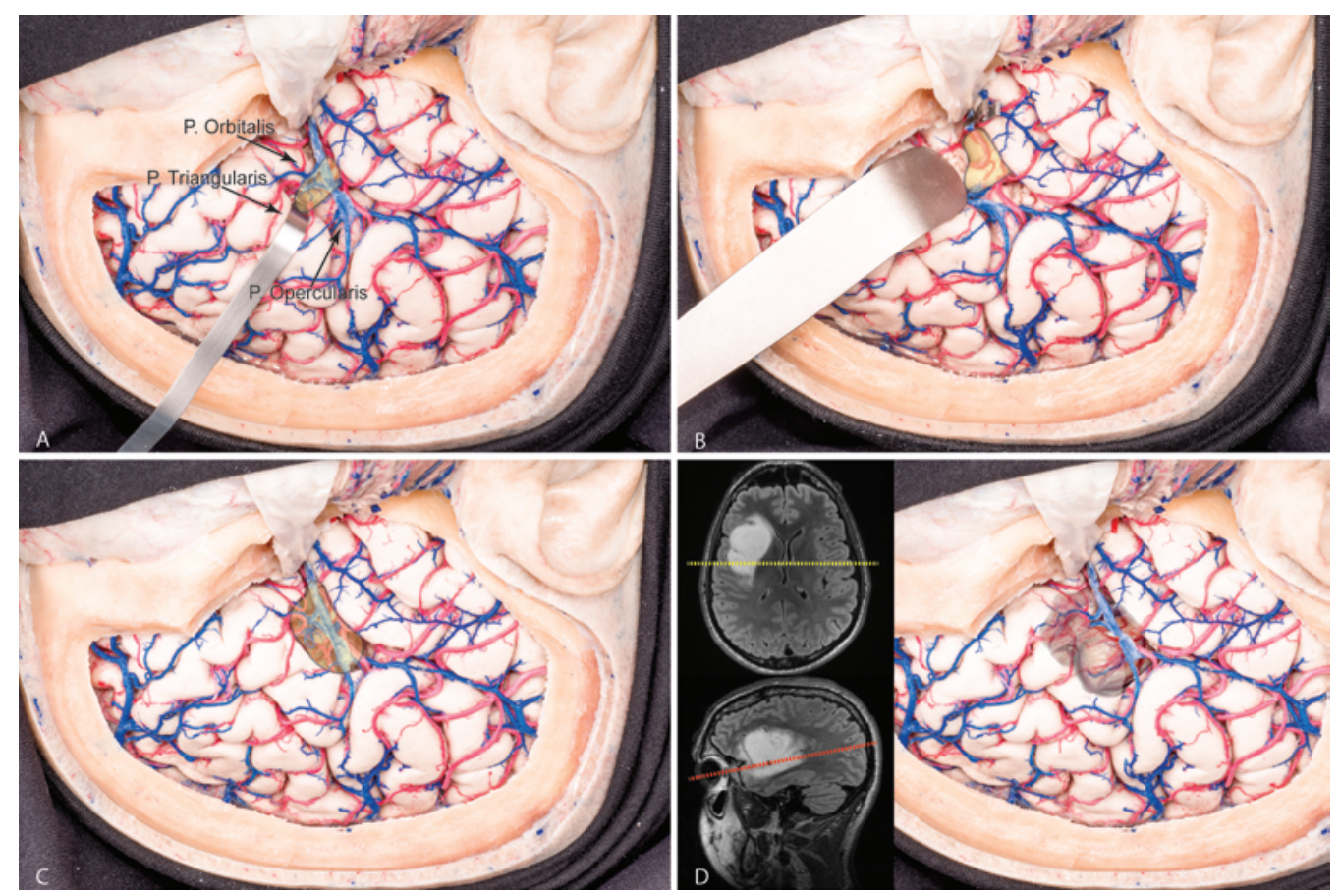

FIG. 5. Surgical simulation of the TS (A), TSVC (B), and TC (C) approaches for the Zone I of the right insula (D). A right-side pterional approach was carried out, and the dura was incised and reflected toward the sphenoid bone. The superficial and deep sylvian cisterns were dissected completely with great care to protect all the vessels. The frontal operculum over Zone I of the insula was retracted to simulate a TS approach (A). Next (B), the superficial sylvian vein was cut and the self-retaining retractors were relocated to simulate the TSVC. The veins were then re-anastomosed, and the frontal operculum was allowed to return to its natural position. The transcortical approach was performed respecting the venous complex and the large veins to the dorsal and orbital aspect of the frontal lobe (C). A typical Zone I insular tumor was photographically fused to the surgical simulation to illustrate the relationship to the cortex before any surgical maneuver was started (D). Axial-superior and sagittal-inferior views of a typical Zone I insular tumor are included to show the relation of the tumor to the Monro line (yellow) and the sylvian line (red). The yellow semitransparent labels in each photograph represent the surgical corridor provided by each approach to the insula. $\mathrm{P}$. $=$ pars. 
ing the TSVC approach and maximal retraction pressure was applied, only $73 \%$ of the total area of the anterior and posterior long gyri could be exposed. The superior periinsular sulcus was not exposed through the TSVC. The precentral gyrus covers the majority of the anterior long gyrus of the insula. Only when the opercular portion of the precentral gyrus was removed, the TC provided more insular exposure than the TSVC.

In Zone III, the TSVC provided greater exposure of the inferior part of the anterior and posterior long gyri, yet the planum temporalis and inferior peri-insular sulcus were only completely exposed through the TC approach (Fig. 7). Heschl's gyrus is a large portion of the posterior aspect of the temporal operculum that blocks the surgical trajectory to the planum temporalis and the posterior long gyrus of the insula. Thus, if Heschl's gyrus is preserved during the TC corridor, the final insular exposure is similar to that of a TSVC with maximal retraction applied uniformly to the superior temporal gyrus. Zone III of the insula, including the inferior peri-insular sulcus, could only be completely exposed when $90 \%$ of the Heschl's gyrus was removed during a TC. Also, if the head was tilted down
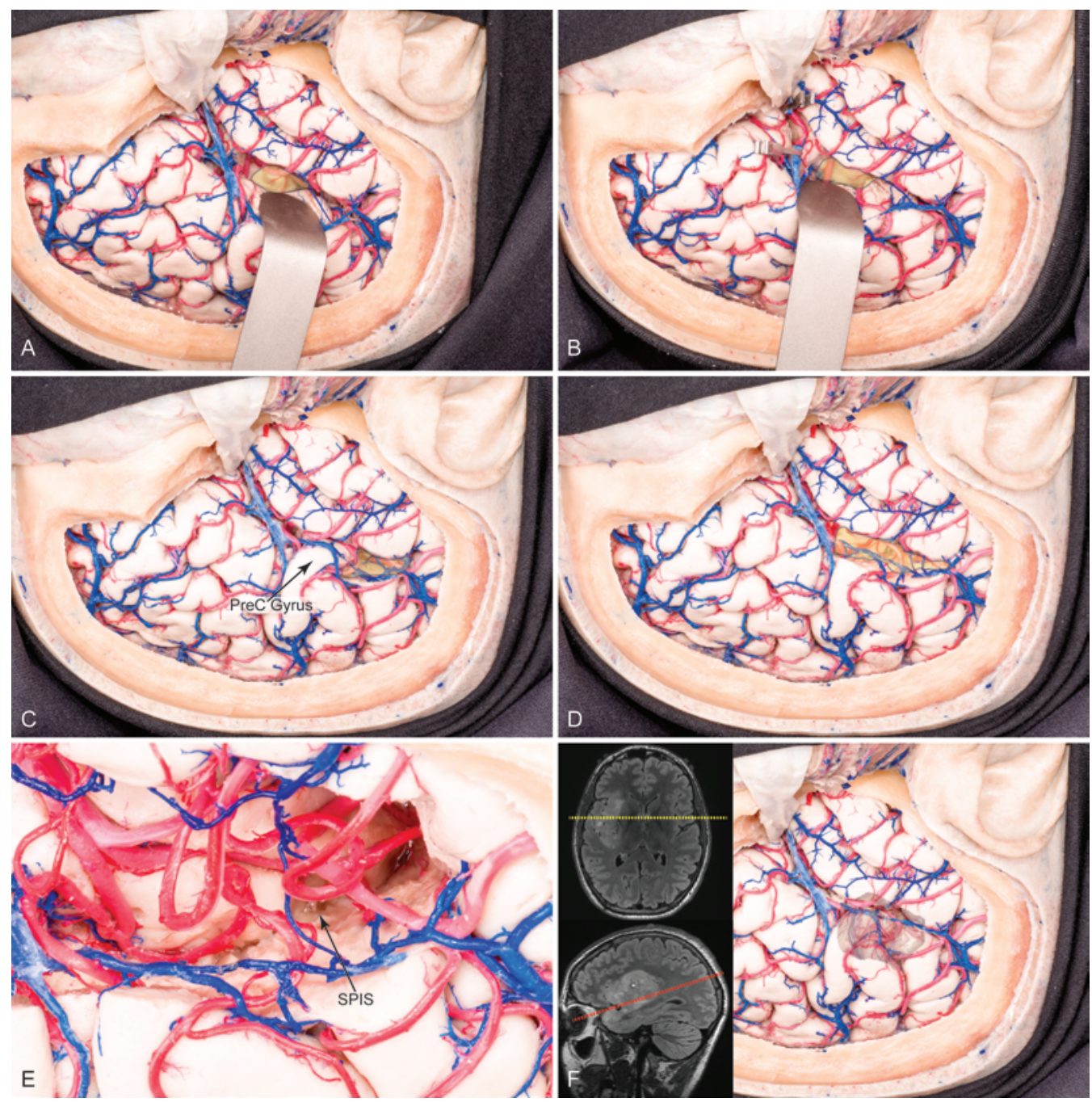

FIG. 6. Surgical simulation of the TS (A), TSVC (B), TC (C), and TC with the precentral gyrus resected (D and E) approaches for Zone II of the insula (F) on the right side. The superficial and deep sylvian cisterns were widely dissected posteriorly with great care to protect all the vessels. The posterior aspect of the frontal operculum and the parietal operculum over Zone II of the insula were retracted to simulate a TS approach (A). Next (B), the main trunk of the superficial sylvian vein was cut and the self-retaining retractors were relocated to simulate the surgical approach with the bridging veins cut. The veins were then re-anastomosed, and the opercula were allowed owed to return to their natural position. The transcortical approach was performed respecting the cortex of the precentral gyrus as well as the venous complex and the large veins to the dorsal aspect of the parietal lobe (C). Next (D and $\mathrm{E})$, the opercular rim of the precentral gyrus was resected to simulate a complete TC approach. A close-up picture of the TC approach to Zone II reveals that the insular cortex of this zone was completely exposed and the superior peri-insular sulcus was also accessible (E). Multiple windows were created between the $\mathrm{M}_{3}$ arteries and the superior sylvian veins, forming multiple flexible corridors to the insular surface. A typical Zone II insular tumor was photographically fused to the surgical simulation to illustrate the relationship to the cortex $(F)$. Axial-superior and sagittal-inferior views of a typical Zone II insular tumor are included in Panel $F$ to show the relation of the tumor to the Monro line (yellow) and the sylvian line (red). The yellow semitransparent labels represent the surgical corridor provided by each approach to the insula. PreC = precentral gyrus; SPIS = superior peri-insular sulcus. 

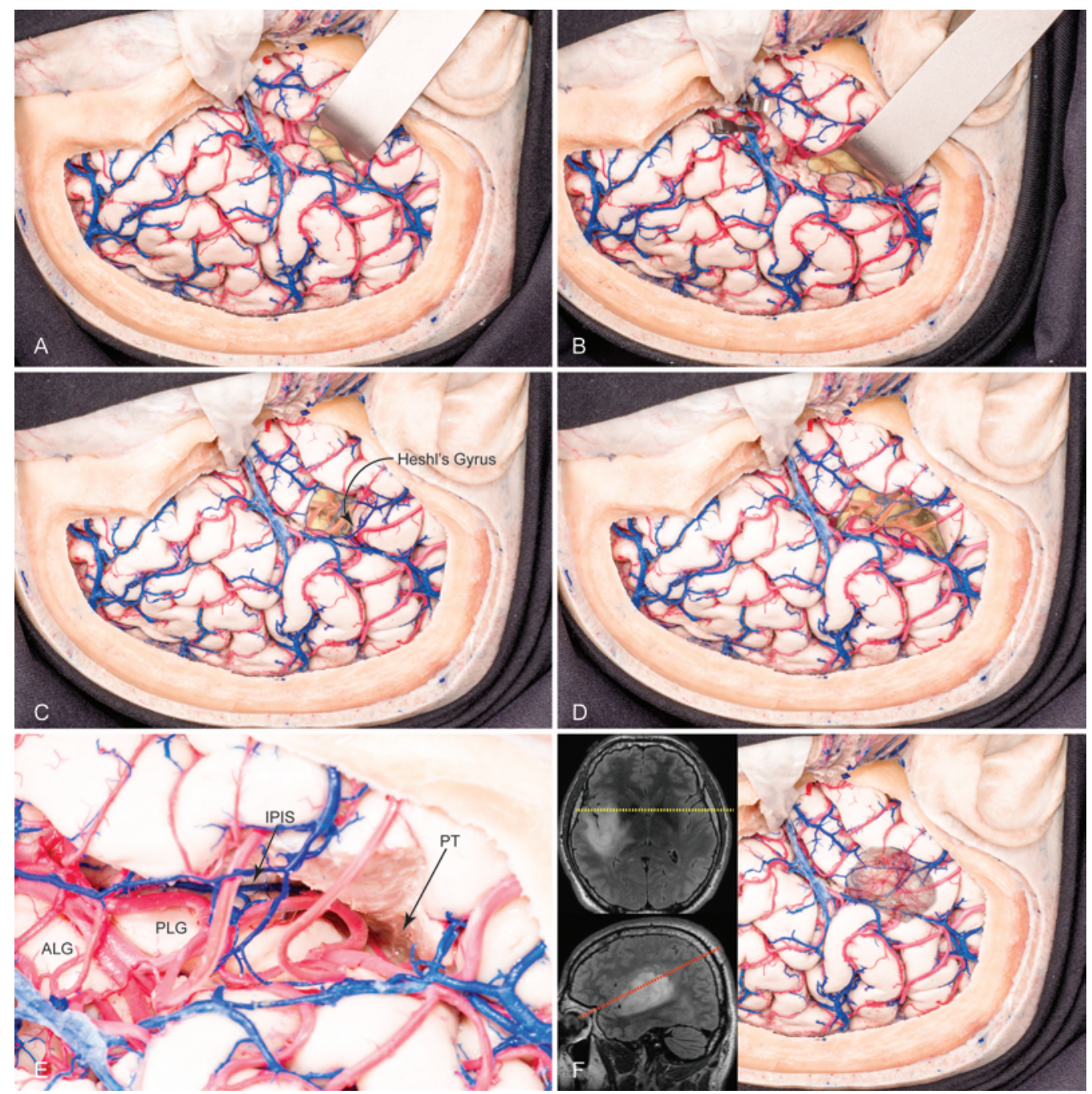

FIG. 7. Surgical simulation of the TS (A), TSVC (B), TC (C), and TC with the Heschl's gyrus resected (D and E) approaches for Zone III of the right insula (F). The superficial and deep sylvian cisterns were completely exposed with great care to protect all the vessels transitioning to the cortex. The posterior segment of the temporal operculum over Zone III of the insula was retracted to simulate a TS approach (A). Following this, the superficial sylvian vein was cut and the self-retaining retractors were reoriented to simulate the surgical approach with the bridging veins cut (B). The veins were then re-anastomosed, and the temporal operculum was allowed to return to its original position. The TC approach was performed next, preserving both Heschl's gyrus and the sylvian venous complex (C). Following this, Heschl's gyrus was resected for a complete TC approach to Zone III of the insula (D and E). A close-up photograph of the TC exposure of the insula revealed complete exposure of the Zone III cortex as well as the planum temporale and inferior peri-insular sulcus (E). The large veins to the dorsal aspect of the temporal and occipital lobes and the Labbé complex as well as the transitioning $\mathrm{M}_{4}$ branches were preserved during all the transcortical dissection. These vessels formed multiple corridors to access the surface of the insula. A typical Zone III insular tumor was photographically fused to the surgical simulation to illustrate the relationship to the cortex before starting the surgical simulation $(F)$. Axial-superior and sagittalinferior views of a typical Zone III insular tumor were included to illustrate the relation of the tumor to the Monro line (yellow) and the sylvian line (red). The yellow semitransparent labels represent the surgical corridor provided by each approach to the insula. ALG = anterior long gyrus; IPIS = inferior peri-insular sulcus; PLG = posterior long gyrus; $\mathrm{PT}$ = planum temporale.

$10^{\circ}-20^{\circ}$ (Trendelenburg or turning down the back rest of the surgical table), the long gyri could be further dissected toward Zone II.

In Zone IV, cutting the bridging veins (TSVC) did not significantly increase insular exposure during a TS corridor, which was completely exposed only through the TC corridor (Fig. 8). The TS corridor exposed the anterior portion of the anterior long gyrus and the insular apex. A large bridging vein limited insular exposure in more than $50 \%$ of specimens. Exposure of the inferior peri-insular sulcus was achieved by cutting this vein (TSVC), applying maximal retraction to the superior temporal gyrus, and tilting the head upward $20^{\circ}$. However, the TC provided more insular exposure than the TSVC. The inferior periinsular sulcus in Zone IV and the insular portion of the planum polaris were completely exposed through the TC corridor.

\section{Venous Drainage}

The venous drainage patterns were carefully studied to 

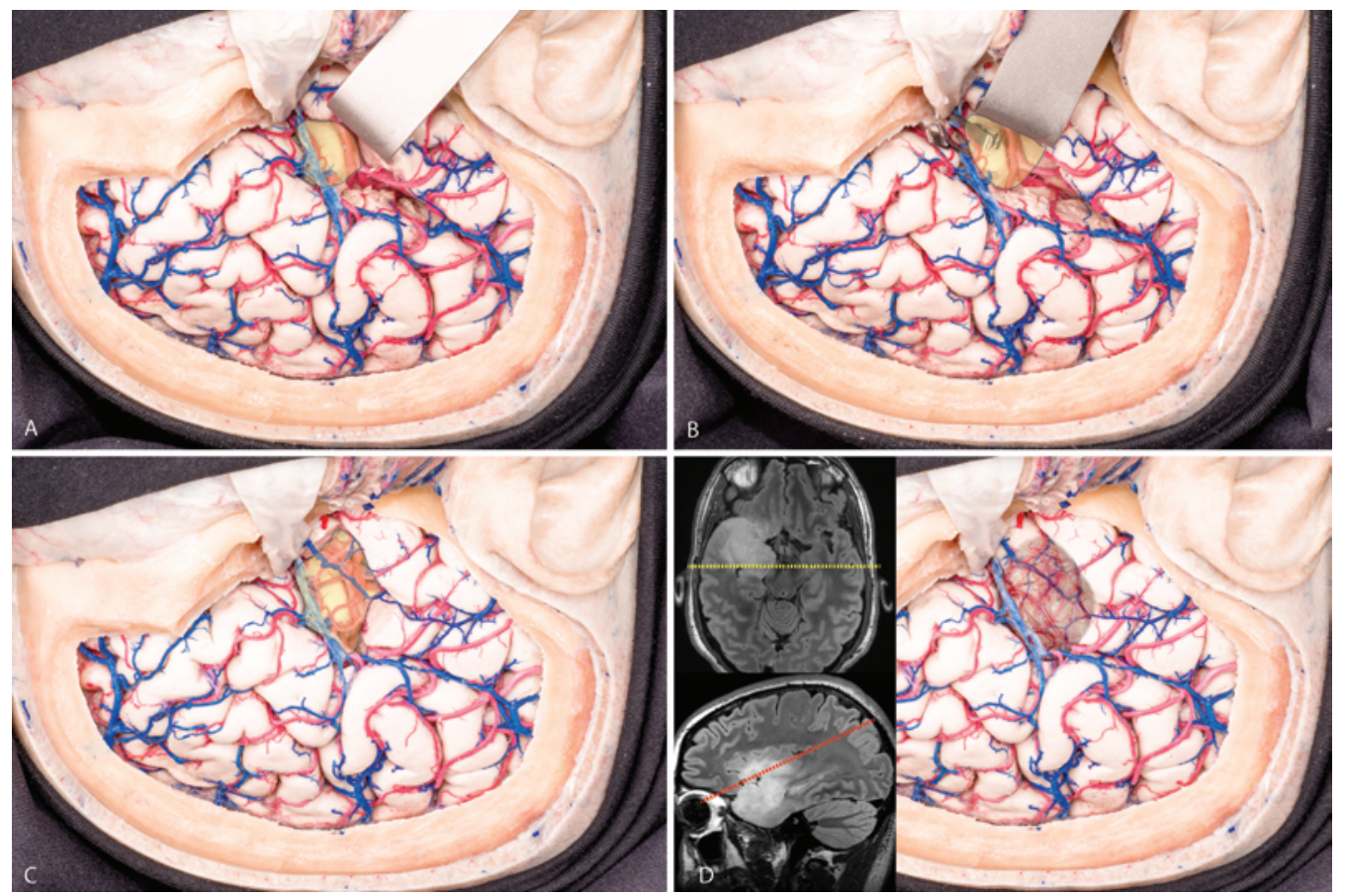

FIG. 8. Surgical simulation of the TS (A), TSVC (B), and TC (C) approaches for Zone IV of the right insula (D). The superficial and deep sylvian cisterns were completely dissected with great care to preserve all the transiting vessels. The temporal operculum over Zone IV was retracted to simulate a TS approach (A). Following this $(B)$, the superficial sylvian vein was cut and the selfretaining retractors were reoriented to simulate the surgical approach with the bridging veins cut. Next, the veins were re-anastomosed, and the temporal operculum was allowed to return to its natural position. The TC approach was performed respecting the venous complex and the large veins to the dorsal aspect of the temporal lobe and the temporal pole (C). Numerous arteriovenous windows were created after the cortical resection and used to access the insular surface. A typical Zone IV insular tumor was photographically fused to the surgical simulation exposure to show the relationship to the cortex before any surgical maneuver was started (D). Axial-superior and sagittal-inferior views of a typical Zone I insular tumor are included to show the relation of the tumor to the Monro line (yellow) and the sylvian line (red). The yellow semitransparent labels represent the surgical corridor provided by each approach to the insula.

determine their dominance in the venous outflow of the lateral surface of the brain, the number of bridging veins and their relationship to the different zones, and the clustering patterns.

We found bridging veins crossing the sylvian fissure in $70 \%(11 / 16)$ of the studied specimens. A dominant drainage pattern was found in $87 \%(14 / 16)$ of cases, where the number and size of veins draining anteriorly was greater than the number and size draining to both the Labbé complex and the superior sagittal sinus. Good alternative outflow was identified in $50 \%(8 / 16)$ of the dominant venous patterns. In $30 \%$ of specimens, there were dominant bridging veins with poor collateral drainage. Moreover, venous clustering around the premotor and prefrontal cortex draining the lower part of the lateral surface of the frontal lobe was found in 56\% (9/16) of the specimens.

\section{Surgical Landmarks}

The observational and descriptive analysis of the surgical anatomy of the pterional transsylvian approach provided 3 key relationships that were consistent: the relationship between the middle cerebral artery running in the central sulcus of the insula $\left(\mathrm{M}_{2}\right)$ and the rolandic artery $\left(\mathrm{M}_{4}\right)$; the relationship between the superior segment of the squamosal suture and the sylvian fissure; and the localization of the foramen of Monro in relation to the external acoustic meatus at the surface of the skull.

There were 2-4 trunks of the $M_{2}$ that branched into 12 $\pm 2 \mathrm{M}_{3}$ branches on the insular surface. In all our specimens, the $\mathrm{M}_{2}$ branches running through the central sulcus of the insula became the precentral (rolandic) artery, feeding the precentral and postcentral gyri. In $81 \%$ of cases $(13 / 16)$, this artery remained at the surface of the operculum. However, in $18 \%(3 / 16)$ of cases, the $\mathrm{M}_{3}$ component of the artery running in the central sulcus of the insula ran in the depth of the precentral sulcus of the brain and became superficial at $0.8 \pm 0.2 \mathrm{~cm}$ from the opercular rim.

The external acoustic meatus was easily identified in all surgical simulations and used as a landmark to infer the position of the foramen of Monro and, therefore, the division of the insula into anterior (I+IV) and posterior (II+III) zones before the craniotomy was done. The foramen of Monro was $1.9 \pm 0.26 \mathrm{~cm}$ anterior and $4.42 \pm 0.6 \mathrm{~cm}$ cranial to the external acoustic meatus.

The squamosal suture was a reliable landmark to infer the position of the sylvian fissure, which defined the surgical division of the insula into superior (I+II) and inferior $(\mathrm{III}+\mathrm{IV})$ zones. The superior portion of the squamosal suture, from the pterion anteriorly to its major inferior bend posteriorly corresponded to the sylvian fissure in the ma- 
jority of specimens (14/16) and was inferior to the sylvian fissure in only 1 specimen (8\%).

\section{Discussion}

This study shows that the TC corridor provides the greatest access to lesions, such as gliomas, within the insular region. The evidence is that even when the comparison between TC, TS, and TSVC is limited to the confines of the insula, the TC still provides the best overall surgical exposure, window, and freedom. In our dissections, the degree of corticotomy in Zones II and III provided significantly different surgical profiles. We found that the value of cutting the bridging veins during a TS approach was maximal in Zone III, where it provided a significant increase in insular exposure. From our observations of the venous drainage of the lateral surface of the brain, we identified a venous pattern that would make the TSVC unsafe in 30\% of cases due to the existence of large dominant sylvian bridging veins with poor alternative outflow to the Labbé or superior sagittal sinus complex. Also, venous clustering at the pars triangularis, opercularis and motor cortex was observed in $56 \%$ of specimens $(9 / 16)$, further limiting the transsylvian corridor to Zones I and II.

The present study suggests that the optimal surgical strategy to obtain maximal exposure for an insular glioma requires careful assessment of each patient's tumor location within the insula. Determining the tumor location in relation to the different zones of the insula allows for preoperative estimation of the extent of tumor resection ${ }^{13}$ and anticipation of the surgical approach that will be required once the cortex is exposed. If the tumor is limited at the sylvian line in Zones I and IV (anterior), our data suggest that the tumor could be sufficiently exposed through a transsylvian approach. However, if the tumor is located in Zones II and III (posterior), using a transsylvian approach may require splitting the sylvian fissure completely and using both extensive retraction and venous sacrifice. This is especially important if the tumor invades into Zone III. However, if the tumor is located either in the periphery of the sylvian line or expands to or beyond the peri-insular sulci, only the transcortical approach will provide a sufficient surgical profile to attempt maximal resection, regardless of the zone. In such cases, direct cortical and subcortical mapping will determine the trajectory and degree of resection in each particular patient.

Whereas cutting the sylvian bridging veins during TS might seem reasonable, our results suggest that such an option could entail serious venous drainage problems in $30 \%$ of cases. Also, we identified clustering of veins in the opercula in more than half of our specimens. Regardless of the overall venous pattern, we consider that a cluster of bridging veins drainingmost of the blood from the inferior frontal gyrus and inferior parietal lobule is, in itself, a limitation to the TSVC. In his study on the cerebral veins, Seeger identified that the direction of the venous blood flow at the lateral surface of the brain was toward the sylvian fissure in $56 \%$ of cases, followed by the Labbé complex and the bridging veins to the superior sagittal sinus. ${ }^{16}$ This finding adds to the important role of the sylvian venous system in the venous outflow of the lateral surface of the brain. In the subgroup of patients who have critical bridging veins that must be preserved, our findings suggest that the TS approach might be insufficient even to expose tumors sitting within the sylvian line, especially in the posterior zones (II+III).

There are several reports on the surgical technique and outcomes of the transsylvian approach to insular tumors. ${ }^{5,9,14,19-21}$ There is common consensus between all groups that large amounts of retraction are required to expose the insula when using the transsylvian corridor, which we have confirmed in our study. However, none of these reports mention the rate in which the sylvian bridging veins were cut. In their series, Lang et al. ${ }^{9}$ and Hentschel and Lang ${ }^{5}$ stated that $2-2.5 \mathrm{~cm}$ of retraction was required to expose the insular tumor. However, the authors did not mention the standard deviation of their measurements, whether they found significant differences in the different parts of the opercula or if the measure referred to the retraction length to one operculum or the distance between opercula after splitting the sylvian fissure. We addressed this uncertainty in our study by measuring the maximal retraction length that could be applied to each operculum without damaging the venous system. In all our specimens, we could not apply $2 \mathrm{~cm}$ of retraction length without cutting the bridging veins. Also, when preserving the venous system (TS approach) the insular exposure was limited and significantly inferior to that of the TSVC and TC. On the basis of these findings, we suspect that in the majority cases in which large insular tumors were described as being successfully removed through a TS approach, the approach was actually TSVC. Although a TSVC corridor would be an acceptable option for those cases in which the venous bridging veins can be sacrificed (70\%) and the insular tumor is within the confines of the peri-insular sulci, we find a major limitation of this strategy in the remaining $30 \%$ of cases, in which a TC approach would potentially overcome this limitation.

Previous studies have shown that TC approaches to subcortical lesions are safe., ${ }^{3,5,13,14}$ Furthermore, our group has reported the first large surgical experience for insular tumors using the TC approach. ${ }^{13}$ Our data suggest that the TC approach provides a better surgical profile than the other options. In fact, the maximum difference in insular exposure between the TC and the TSVC was observed in the posterior insular zones, where the TS corridor is severely limited by the narrow sylvian cistern. However, the surgical profile of the TC approach to the posterior zones is highly dependent on brain mapping and therefore impossible to predict before surgery. Although cortical sacrifice is inherent to the TC corridor, direct cortical and subcortical stimulation during awake surgery allows the neurosurgeon to identify function and work around eloquent areas. In contrast, when dissecting the sylvian cistern, especially the posterior portion, the neurosurgeon has to perform meticulous dissection of critical neurovascular structures in a very narrow corridor. In a study of surgical approaches to temporal tumors in 235 patients, Schramm et al. ${ }^{14,15}$ reported that the TS approach was associated with the highest combined rate of complications, which could be caused by inadvertent subpial dissection or transection of an insular artery. Using a transsylvian approach to in- 
sular tumors, Hentschel and Lang reported a postoperative speech complication rate of $30 \%$, which they attributed to transient ischemia related to both retraction and arterial dissection. These results are congruent with the evidence of our morphometric analysis, where large amounts of retraction over the opercula, including the pars opercularis and the precentral gyrus, are required for maximal insular exposure.

The transcortical approach to the insula uses direct cortical and subcortical stimulation to assess and preserve cortical function. While the anatomical location of cortical function is variable, there are general patterns of functional anatomy that may guide surgical planning. In Zone I, the transcortical approach transgressed $60 \%$ of pars orbicularis and opercularis, and $20 \%$ of pars triangularis. In the dominant hemisphere, language function is classically described in Brodmann Areas 44 and 45 (also known as Broca's area). In our experience, only the posterior aspect of pars opercularis of the dominant hemisphere is highly involved in speech production. ${ }^{8}$ However, in those patients with tumors affecting pars opercularis, transcortical resection under constant cortical mapping may be safe. ${ }^{8}$ In Zone II, the opercular segment of the precentral gyrus, which may account for the contralateral motor control of the face, sits on top of the anterior long gyrus of the insula. Our data show that if the opercular portion of the precentral gyrus is removed, the insular exposure is $146 \%$, and the surgical freedom is $171 \%$ that of TSVC. There is evidence of recovery after resection of the facial motor cortex in the nondominant hemisphere, ${ }^{10}$ which has been congruent with the surgical results published by our group ${ }^{13}$ and that of Duffau et al. ${ }^{4}$ If resection of the facial motor cortex in the dominant hemisphere is attempted, it should be limited to pure facial expression, which will cause transient central facial paralysis. After studying a series of 14 patients who underwent an awake transcortical approach for tumors affecting Zone II of the insula and the inferior parietal lobule, Maldonado et al. identified speech function in the majority of cases. Interestingly, they found high interindividual variability in the anatomical location of language. Therefore, awake cortical and subcortical stimulation must be performed in each case to assess the location of functional cortex and white matter tracts for language, which will further characterize the final transcortical corridor in each patient. ${ }^{11}$ The posterior margin of the transcortical approach in Zone II involved $1 \mathrm{~cm}$ of the postcentral gyrus, which may account for the somatosensory function of the contralateral half of the face. In Zone III, exposure of the posterior long gyrus and planum temporalis requires excision, to some degree, of Heschl's gyrus. When Heschl's gyrus is resected along with the anterior segment of the superior temporal gyrus, the insular exposure becomes $164 \%$ that of the TSVC. The primary auditory cortex, located at the Heschl's gyrus in both hemispheres, receives bilateral afferent sensory signaling from the cochlear and superior olivary nucleus via the inferior colliculus and medial geniculate body of the thalamus. ${ }^{2}$ In a recent study, Javad et al. provided evidence of interhemispheric transcallosal linkage between the auditory areas using diffusion tensor imaging in humans. ${ }^{7}$ Hence, complete resection of Heschl's gyrus in 1 hemi- sphere (e.g., TC to Zone III of the insula) should not cause a perceivable loss in audition. Nonetheless, our experience is congruent with other authors in that resection of the superior temporal gyrus under direct cortical stimulation is safe. ${ }^{4,13}$ Cortical resection to expose Zone III does not involve the angular gyrus, or regions posterior to Heschl's gyri, which, in the dominant hemisphere, may account for Wernicke's area (Brodmann Area 22). When resecting tumors in Zone II and III of the insula, constant direct cortical and subcortical stimulation of the superior temporal gyrus and parietal operculum are performed before any corticectomy to assess for language function, especially in the dominant hemisphere. ${ }^{4}$ The arcuate and middle longitudinal fascicles, and Wernicke's area, which are in the vicinity of Heschl's gyrus, are presumed to be a core part of the language pathway, therefore potentially limiting the transcortical corridor around the primary auditory cortex in the dominant hemisphere. However, the location and composition of such areas and tracts varies considerably in the population. Therefore intraoperative functional mapping becomes essential to guide the resection in each patient.

Although the use of intraoperative navigation is very important in modern neurosurgery, it should not replace anatomical knowledge. In this study, we identified two anatomical landmarks that may be used to infer the location of the insular zones before opening the skull. We found a strong correlation between the division of the insular lobe into anterior and posterior parts and the external acoustic meatus over the skull surface. Also, we found that the superior segment of the squamosal suture could be used to infer the location of the sylvian fissure, which divides the insular lobe into dorsal and ventral segments. Knowledge of these anatomical relations may aid in tailoring the craniotomy to the insular lesion.

Thorough knowledge of the arterial blood supply beyond $\mathrm{M}_{2}$ is critical to preserve function while dissecting an insular tumor. When using microsurgical dissection around the insula, the high power magnification reduces peripheral view. It is crucial to recognize the cortical distribution related to each $\mathrm{M}_{2}$ artery transiting within the middle cerebral artery's candelabra, as it is being dissected. We have found that the $\mathrm{M}_{2}$ artery running over the central sulcus of the insula becomes the rolandic artery in $100 \%$ of our specimens, which is in agreement to the findings reported by Türe et al. in their study on the insular arteries..$^{18}$ Interestingly, we observed that the $\mathrm{M}_{3}$ segment of the rolandic artery runs in the depth of the operculum in $18 \%$ of (3/16) cases, which requires careful dissection when using the TC corridor to approach Zone I or II.

\section{Study Limitations}

Human postmortem surgical simulation provides the best alternative to surgical experimentation because it is safe, provides a very realistic scenario of the human anatomy, and allows for prolonged research time. In our recent publication, ${ }^{1}$ we described our customized surgical simulation method for neurosurgical research. One of the most interesting findings of the study was that there were no differences on the retraction profile between the specimens prepared with our customized embalming formula 
and that of unembalmed (i.e., fresh) cadavers. ${ }^{1}$ This new method overcomes the major limitations for surgical simulation when using classical cadaver processing techniques (brain stiffness and inability to retract), allowing a very realistic surgical simulation with life-like manipulation of the brain. Therefore, the present study uses the most advanced cadaveric neurosurgical research methods to provide information on the surgical profile of the transsylvian and transcortical approaches to the insula. However, several limitations, intrinsic to postmortem research, may limit direct application of our findings to clinical practice. These limitations include the lack of effective brain relaxation; absence of anatomical distortion due to masseffect; and the impossibility to study cortical function. In this study, thorough dissection of arachnoid adhesions, evacuation of cerebrospinal fluid, and opercular retraction allowed splitting the sylvian fissure widely. However, brain relaxation techniques such hyperventilation and the use of brain osmotic agents could not be applied therefore limiting the surgical results of the study. The mass effect related to insular lesions may cause substantial anatomical distortion and should be considered in the preoperative planning. However mass effect is highly variable and casespecific. We used specimens without known brain pathology to maximize statistical power and internal validity when comparing surgical approaches. Direct assessment of cortical function is an inherent limitation of postmortem research and may limit the clinical application of this study. However, the anatomical location of brain function is specific to each patient and situation (e.g., neural plasticity) and therefore the impact of cortical function location to the transcortical approach should be assessed in every case using intraoperative stimulation mapping of the language function.

\section{Conclusions}

There are 3 surgical options to approach insular gliomas: the transsylvian approach, the transsylvian approach with the bridging veins cut, and the transcortical approach (referred to in this paper as TS, TSVC, and TC, respectively). Overall, the TC approach provided better insula access than the TS approach. Although in some circumstances the TC provides similar surgical exposure and surgical freedom to that of the TSVC, cutting bridging veins may be unsafe in $30 \%$ of patients. Cortical and subcortical mapping is critical before and during the transcortical approach to the posterior zones (Zones II and III), as the facial motor and somatosensory functions (Zone II) and the language pathways (Zone III) are involved. The greatest insular exposure in Zones II and III requires resection of the precentral gyrus and superior temporal gyrus. While the TC and TSVC provide equivalent access to the insula in Zones I and II, a TC approach may be needed to maximize access Zones III and IV (inferior). Thus, preoperative assessment using radiological imaging and our insula classification scheme is useful in guiding the surgical planning. This study demonstrates that the TC approach may be superior to the TSVC approach in accessing gliomas that, while primarily within the insula, extend to and beyond the peri-insular sulcus. However, neurosurgeons may also consider the TS and TSVC approaches for small to moderate size lesions located within the confines of the insula. Selecting the surgical option providing the greatest insular exposure should also help reduce surgical morbidity.

\section{Acknowledgments}

We wish to thank all individuals and their families who, without any other interest than that of research, willed their bodies to achieve these results. This study would not have been possible without their contribution and therefore to them we dedicate the present report. The authors would like to also thank Annette Molinaro for her contribution to the statistical analysis and Simar Singh for the medical illustration included in the present study.

\section{References}

1. Benet A, Rincon-Torroella J, Lawton MT, González Sánchez JJ: Novel embalming solution for neurosurgical simulation in cadavers. J Neurosurg 120:1229-1237, 2014

2. Crippa A, Lanting CP, van Dijk P, Roerdink JB: A diffusion tensor imaging study on the auditory system and tinnitus. Open Neuroimaging J 4:16-25, 2010

3. Duffau H: A personal consecutive series of surgically treated 51 cases of insular WHO Grade II glioma: advances and limitations. J Neurosurg 110:696-708, 2009

4. Duffau H, Capelle L, Lopes M, Faillot T, Sichez JP, Fohanno $\mathrm{D}$ : The insular lobe: physiopathological and surgical considerations. Neurosurgery 47:801-811, 2000

5. Hentschel SJ, Lang FF: Surgical resection of intrinsic insular tumors. Neurosurgery 57 (1 Suppl):176-183, 2005

6. Ius T, Pauletto G, Isola M, Gregoraci G, Budai R, Lettieri $\mathrm{C}$, et al: Surgery for insular low-grade glioma: predictors of postoperative seizure outcome. J Neurosurg 120:12-23, 2014

7. Javad F, Warren JD, Micallef C, Thornton JS, Golay X, Yousry T, et al: Auditory tracts identified with combined fMRI and diffusion tractography. Neuroimage 84:562-574, 2014

8. Rolston JD, Englot DJ, Benet A, Li J, Cha S, Berger MS: Frontal operculum gliomas: language outcome following resection. J Neurosurg 122:725-734, 2015

9. Lang FF, Olansen NE, DeMonte F, Gokaslan ZL, Holland EC, Kalhorn C, et al: Surgical resection of intrinsic insular tumors: complication avoidance. J Neurosurg 95:638-650, 2001

10. LeRoux PD, Berger MS, Haglund MM, Pilcher WH, Ojemann GA: Resection of intrinsic tumors from nondominant face motor cortex using stimulation mapping: report of two cases. Surg Neurol 36:44-48, 1991

11. Maldonado IL, Moritz-Gasser S, de Champfleur NM, Bertram L, Moulinié G, Duffau H: Surgery for gliomas involving the left inferior parietal lobule: new insights into the functional anatomy provided by stimulation mapping in awake patients. J Neurosurg 115:770-779, 2011

12. Potts MB, Chang EF, Young WL, Lawton MT: Transsylviantransinsular approaches to the insula and basal ganglia: operative techniques and results with vascular lesions. Neurosurgery 70:824-834, 2012

13. Sanai N, Polley MY, Berger MS: Insular glioma resection: assessment of patient morbidity, survival, and tumor progression. J Neurosurg 112:1-9, 2010

14. Schramm J, Aliashkevich AF: Surgery for temporal mediobasal tumors: experience based on a series of 235 patients. Neurosurgery 60:285-295, 2007

15. Schramm J, Aliashkevich AF: Surgery for temporal mediobasal tumors: experience based on a series of 235 patients. Neurosurgery 62 (6 Suppl 3):1272-1282, 2008

16. Seeger W: Microsurgery of Cerebral Veins. Vienna: Springer, 2000 
17. Skrap M, Mondani M, Tomasino B, Weis L, Budai R, Pauletto G, et al: Surgery of insular nonenhancing gliomas: volumetric analysis of tumoral resection, clinical outcome, and survival in a consecutive series of 66 cases. Neurosurgery 70:1081-1094, 2012

18. Türe U, Yaşargil MG, Al-Mefty O, Yaşargil DC: Arteries of the insula. J Neurosurg 92:676-687, 2000

19. Vanaclocha V, Sáiz-Sapena N, García-Casasola C: Surgical treatment of insular gliomas. Acta Neurochir (Wien) 139:1126-1135, 1997

20. Wang P, Wu MC, Chen SJ, Xu XP, Yang Y, Cai J: Microsurgery resection of intrinsic insular tumors via transsylvian surgical approach in 12 cases. Cancer Biol Med 9:44-47, 2012

21. Yaşargil MG, von Ammon K, Cavazos E, Doczi T, Reeves JD, Roth P: Tumours of the limbic and paralimbic systems. Acta Neurochir (Wien) 118:40-52, 1992

\section{Disclosure}

Dr. Lawton receives royalties related to the "Lawton Bypass set" from Mizuho instruments.

\section{Author Contributions}

Conception and design: Benet, Hervey-Jumper, Lawton, Berger. Acquisition of data: Benet. Analysis and interpretation of data: all authors. Drafting the article: Benet. Critically revising the article: all authors. Reviewed submitted version of manuscript: all authors. Approved the final version of the manuscript on behalf of all authors: Benet. Statistical analysis: Benet, Hervey-Jumper, González Sánchez. Administrative/technical/material support: Benet, González Sánchez, Lawton, Berger. Study supervision: Benet, Lawton, Berger. Surgical simulations/experiments: Benet.

\section{Supplemental Information \\ Companion Paper}

Hervey-Jumper SL, Li J, Osorio JA, Lau D, Molinaro AM, Benet A, et al: Surgical assessment of the insula. Part 2: validation of the Berger-Sanai zone classification system for predicting extent of glioma resection. DOI: 10.3171/2015.4.JNS1521.

\section{Correspondence}

Arnau Benet, Department of Neurosurgery, University of California San Francisco, 505 Parnassus Ave., Rm. M779, San Francisco, CA 94143. email: arnaubenet@gmail.com. 